\title{
The Impact of Radical Prostatectomy on the Survival of Patients with Carcinosarcoma of the Prostate
}

\author{
Jue Wang ${ }^{*}$, Fenwei Wang ${ }^{2}$ \\ ${ }^{1}$ Department of Internal Medicine, Section of Oncology-Hematology, University of Nebraska Medical Center, Omaha, USA; ${ }^{2}$ Department \\ of Internal Medicine, Creighton University Medical School, Omaha, USA. \\ Email: juewang@unmc.edu
}

Received July $4^{\text {th }}, 2011$; revised August $6^{\text {th }}, 2011$; accepted August $25^{\text {th }}, 2011$.

\begin{abstract}
Background: The objective of this study was to evaluate the impact of radical prostatectomy on carcinosarcoma of the prostate. Methods: Patients diagnosed with carcinosarcoma of the prostate from 1977 through 2007 were identified in the Surveillance, Epidemiology, and End Results (SEER) database. Outcomes were examined with Kaplan-Meier survival analysis and Cox models. The association between clinical and demographic characteristics and survival of carcinosarcoma of the prostate was examined. Results: A total of fifty-four cases of histology confirmed carcinosarcoma of the prostate were identified. Median age of the patients was 74 years (range 28 - 94). Of the patients with a known tumor stage, all but one patient had a locally advanced or distant stage; all of the patients with known histology grade had poorly or undifferentiated histology. Twenty (37\%) patients underwent transurethral resection only and Fourteen (25.9\%) patients underwent radical prostatectomy. Eight (14\%) patients received radiation therapy in combination with surgery. The median cancer specific survival was 16 months (95\% CI 0 - 32 months). 1-, 3-, and 5- year cancer specific survival rate were $55.1 \%, 32.3 \%$ and $21.1 \%$. In a multivariate analysis, radical prostatectomy was found to be a significant prognostic factor for cancer-specific survival. Conclusions: Carcinosarcoma of the prostate commonly occurs in older patients and associated with aggressive disease and poor prognosis. Radical prostatectomy is the only treatment modality that significantly improves survival. Emphasis on early diagnosis and detection and multimodality therapy of this disease is needed to improve the outcome for patients with this malignancy.
\end{abstract}

Keywords: Carcinosarcoma of the Prostate, Surveillance, Epidemiology, End Results (SEER), Prostatectomy, Cancer-Specific Survival (CSS)

\section{Introduction}

Carcinosarcoma of the prostate is a rare and aggressive tumor characterized by the coexistence of malignant high grade epithelial and mesenchymal components [1]. Less than 100 cases of prostate carcinosarcoma have been reported in the literature, most often as single case report or limited series [2-10] with focus on histopathological characteristics. Microscopically, carcinosarcomas are biphasic tumors made of an intimate admixture of carcinomatous and sarcomatous components with abrupt or gradual transition from one to the other. In most cases, the epithelial component consists of high grade adenocarcinoma with possible epidermoid and/or glandular differentiation, while the heterologous component consists of chondrosarcoma, malignant fibrous histiocytoma, osteosarcoma, leiomyosarcoma, fibrosarcoma or rhabdomyosarcoma [7-10]. The epithelial component is cytokeratin, PSA- and PAP-positive. In a minority of cases, the spindle cell element expresses cytokeratin, but PSA and PAP are rarely positive [5]. Clinically, these patients tend to be older, and present with advanced stage [1-6]; in roughly half of cases have a history of prostatic adenocarcinoma treated by radiation $[3,4]$. The tumors commonly produce bladder outlet obstruction and often require repeated transurethral resection of the prostate (TURP) to control local symptoms. Serum PSA levels are usually lower than expected for the tumor volume.

The histogenesis of prostate carcinosarcoma is not completely understood, and various interpretations have been proposed for the heterogenous components of this tumor [11-17]. Some investigators suggested that these 
tumors develop as a result of undifferentiated, totipotential neoplastic cells that undergo multiple pathways of terminal differentiation into either mesenchymal or epithelial elements. This theory is supported by the presence of epithelial markers (cytokeratin or EMA) in mesenchymal areas and the presence of ultrastructural features (desmosomes or tonofilaments) of epithelial differentiation in sarcomatoid elements [11]. Others believe that in cases where different components share no common features on immunohistochemical and electron microscopic examinations, carcinosarcomas might be the result of true "collision" tumors, where both malignant epithelial and mesenchymal components arise independently from each other [16]. Several investigators evaluated clonality in both malignant epithelial and mesenchymal elements using genetics or molecular techniques. The tumor cells from both tumor components showed monoclonality and clonal identity in all cases studied, suggesting a monoclonal origin [13-15]. Other theories include carcinosarcoma might developed as a equence of conmetaplastic chondromatous response which subsequently became malignant in patients with primary adenocarcinoma of the prostate $[17,18]$; or originate from the mesodermal vestige within the prostate [19].

Due to the rarity of this malignancy, current knowledge about carcinosarcoma of the prostate is limited to small retrospective case series and case reports. The clinical significance and biologic behavior of this subtype of prostate cancer remains poorly understood. To improve our understanding of this tumor, we have undertaken a comprehensive analysis of patients with carcinosarcoma of the prostate identified in the National Cancer Institute's Surveillance, Epidemiology, and End Results (SEER) database.

\section{Methods}

\subsection{Data Source}

Surveillance, Epidemiology, and End Results (SEER) databases included patient records from multiple sites across the United States. The database was designed to reflect overall characteristics of the United States, including the variety of racial/ethnic groups, geographical locations. SEER 9, 13, and 17 registries cover approximately $9.5 \%, 13.8 \%$, and $26.2 \%$ of the total US population, respectively [20]. Data for this study were obtained from SEER*Stat public-use data files, available on the internet at the National Cancer Institute web site.

\subsection{Study Population and Variables}

The cases of prostatic carcinosarcoma were extracted from the SEER on the basis of anatomic site (ICD-O-2 codes C61.9) and histologic type (ICD-O code 8980) for those patients first diagnosed and/or treated between January 1977 and December 2007.

Patients' social demographic characteristics (i.e. age, race/ethnicity and marital status) and tumor grade and stage at the time of diagnosis, were determined from the SEER database. SEER general summary stage [21] classifies patients as having local, regional (extension into adjacent tissues or nodal involvement), or distant disease. The World Health Organization's standard grading system was used with four separate categories (well, moderately well, poorly differentiated, and undifferentiated). For prostate cancer cases, SEER database recorded the highest value of PSA tests at the time of diagnosis under the variable named "Tumor Marker 2". In the database, PSA was categorized as a) none, b) positive, c) negative, d) borderline, undetermined whether positive or negative, e) ordered, but results not in chart, f) unknown or no information [21]. According to the available information, we grouped PSA levels into three groups in this study: above normal (positive), normal (negative) or unknown.

\subsection{Statistical Analysis}

Discrete data are reported as frequencies and compared by chi-square and Fisher's exact tests as appropriate. Continuous data are reported as mean \pm SD and compared by student's $t$ test. Cases identified at the time of autopsy or by death certificate only or with more than one primary were excluded from survival analyses. Survival duration was measured by the Kaplan-Meier method [22] and compared by the log rank test. Multivariate survival analyses were carried out using the Cox proportional hazard model. Hazard ratios and 95\% confidence intervals were reported [23]. All other statistical calculations were performed by SPSS 12.0 (Apache Software Foundation 2000). Comparative differences were considered statistically significant when the $\mathrm{p}$ value was $<0.05$.

\section{Results}

\subsection{Patient and Tumor Characteristics}

Between 1977 and 2007, a total of 54 patients with histologically confirmed carcinosarcoma of the prostate were identified in the SEER 17 registries. The median age of these patients at diagnosis was 74 years, with a range of 28 to 94 years. Forty-six patients $(85.8 \%)$ were white, while African American accounted for 5 (9.3\%) patients. Other ethnicities accounted for 3 patients (5.6\%). Details of patient and tumor characteristics of study cohort are included in Table 1.

Of the 36 patients with known AJCC stage, 35 patients presented with either locally advanced or distant stage; all of the 27 patients with known histology grade had 
Table 1. Characteristics of 54 patients with carcinosarcoma of the prostate diagnosed between January 1977 and December 2007.

\begin{tabular}{|c|c|c|}
\hline \multicolumn{2}{|c|}{ Characteristics } & \multirow{2}{*}{$\frac{\text { Total patients N }(\%)}{73( \pm 12)}$} \\
\hline Age groups & Mean $( \pm \mathrm{SD})$ & \\
\hline & $<75 \mathrm{yrs}$ & $29(53.7)$ \\
\hline & $\geq 75 \mathrm{yrs}$ & $25(46.3)$ \\
\hline \multirow[t]{3}{*}{ Race } & Black & $5(9.3)$ \\
\hline & White & $46(85.8)$ \\
\hline & Others & $3(5.6)$ \\
\hline \multirow[t]{3}{*}{ Married } & Yes & $36(66.7)$ \\
\hline & No & $15(27.8)$ \\
\hline & Unknown & $3(5.5)$ \\
\hline \multirow[t]{4}{*}{ Grade } & Moderately-differentiated & $3(5.6)$ \\
\hline & Poorly-differentiated & $12(22.2)$ \\
\hline & Undifferentiated & $12(22.2)$ \\
\hline & Unknown & $27(50.0)$ \\
\hline \multirow[t]{3}{*}{ AJCC Stage } & $<\mathrm{T} 3$ & $1(1.9)$ \\
\hline & $\geq \mathrm{T} 3$ & $35(64.8)$ \\
\hline & Unknown & $18(33.3)$ \\
\hline \multirow[t]{3}{*}{ SEER Stage } & Local/regional & $21(38.9)$ \\
\hline & Distant & $13(24.1)$ \\
\hline & Unknown & $20(37.0)$ \\
\hline \multirow[t]{3}{*}{ PSA } & Above normal & $12(22.2)$ \\
\hline & Normal & $5(9.3)$ \\
\hline & Unknown & $37(68.5)$ \\
\hline \multirow[t]{2}{*}{ Year of diagnosis } & $1977-1994$ & $19(35.2)$ \\
\hline & $1995-2007$ & $35(64.8)$ \\
\hline \multirow[t]{4}{*}{ CDS } & Prostatectomy & $14(25.9)$ \\
\hline & TURP & $20(37.0)$ \\
\hline & Surgery NOS & $4(7.4)$ \\
\hline & No & $16(29.6)$ \\
\hline \multirow[t]{2}{*}{ Radiation } & Yes & $17(31.5)$ \\
\hline & No & $37(68.5)$ \\
\hline
\end{tabular}

$\mathrm{CDS}=$ cancer directed surgery; PSA = prostate specific antigen; American Joint Committee on Cancer (AJCC); Transurethral resection of the prostate (TURP).

poorly or undifferentiated histology.

\subsection{Treatment}

Most of patients (70.4\%) were treated with cancer directed surgery (CDS): twenty patients $(37.0 \%)$ underwent transurethral resection of the prostate (TURP) only and fourteen $(25.9 \%)$ patients underwent radical prostatectomy. Seventeen $(17.9 \%)$ of patients received radiation therapy, among them, nine patients received radiation therapy as primary therapy; eight patients received radiation in combined with cancer directed surgery (One patient received radiation before radical prostatectomy; five patients received radiation after radical prostatectomy; one patients received radiation after TURP; one patient received radiation before and after radical prostatectomy) (Table 1).

\subsection{Survival}

All patients had active follow-up (range 0 - 127 months); 48 of $54(88.9 \%)$ patients died during the follow-up period. Figure 1(a) presents Kaplan-Meier survival curves for overall survival of entire cohort. The median overall survival was 13 months (95\% CI 8 - 18 months) (Figure 1(a)). Figure 1(b) presents Kaplan-Meier survival curves for cancer specific survival of entire cohort. The median cancer specific survival was 16 months $(95 \%$ CI 0 - 32 months) (Figure 1(b)).

Table 2 presents the 1-, 3-, and 5- year cancer specific survival rate according to patients and tumor characteristics. Figure 1(c) presents Kaplan-Meier survival curve according to therapy. There is a significant difference of cancer specific survival among those who underwent prostatectomy and those did not $(\mathrm{p}<0.001)$.

Table 3 presents the result of multivariate survival analyses using Cox proportional hazard model. After adjusting for the demographic, clinical and treatment related factors, only prostatectomy was identified as an independent factor associated with cancer specific sur$\operatorname{vival}(\mathrm{HR}=0.175 ; 95 \% \mathrm{CI} 0.064-0.478 ; \mathrm{p}=0.001)$.

\section{Discussion}

Although a single center study reported an incidence rate of $0.79 \%$ [3], the true incidence of carcinosarcoma of the prostate is unknown. The incidence in this population study is much lower than what was reported in single institution studies [4]. This discrepancy likely reflects the fact that single institution studies are usually reported from large, tertiary-care referral centers, where the patients with rare tumors tend to seek second opinion. Current practice pattern may be also contributed to current finding. It was reported $50 \%$ of carcinosarcoma of the prostate cases were found in case of prostate adenocarcinoma previously treated with hormone or radiation therapy $[4,7,24,25]$. Re-biopsies are seldom performed in

Table 2. 1-, 3-, and 5-year cancer specific survival of patients with carcinosarcoma of the prostate according to demographic and clinical characteristics.

\begin{tabular}{cccc}
\hline Charactistics & \multicolumn{3}{c}{ Cancer Specific Survival Rate (\%) } \\
\hline & 1-year & 3-year & 5-year \\
Overall patients & 55.1 & 33.3 & 21.2 \\
Prostatectomy & & & \\
No & 42.1 & 17.0 & 12.8 \\
Yes & 92.3 & 75.5 & 43.2 \\
\hline
\end{tabular}




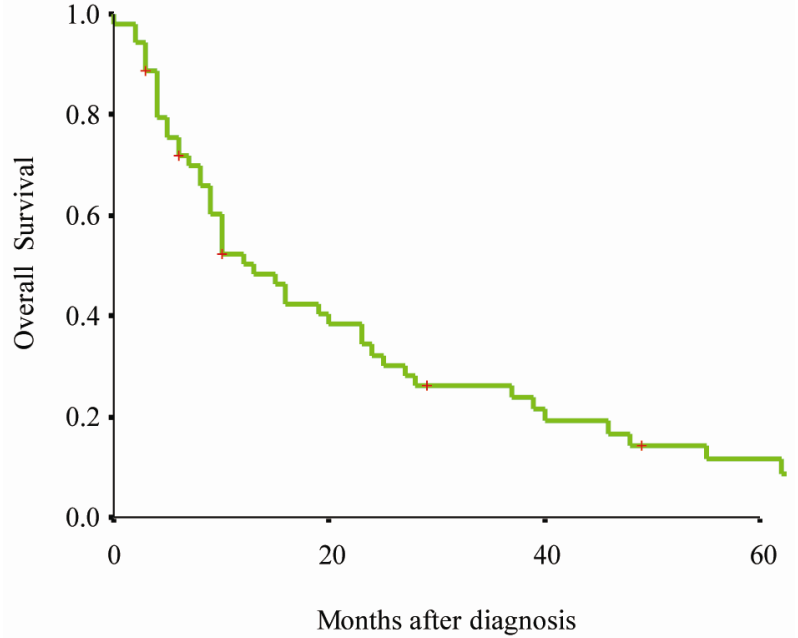

(a)

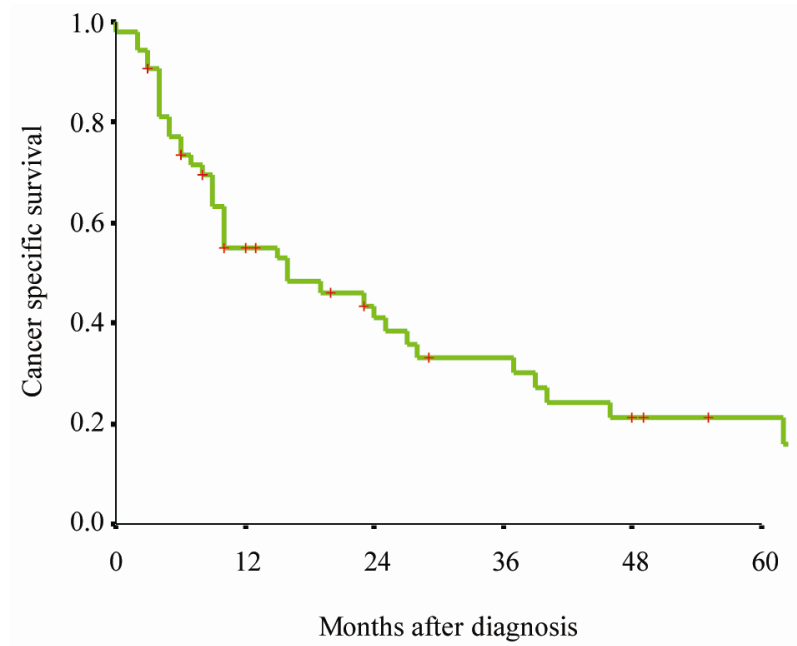

(b)

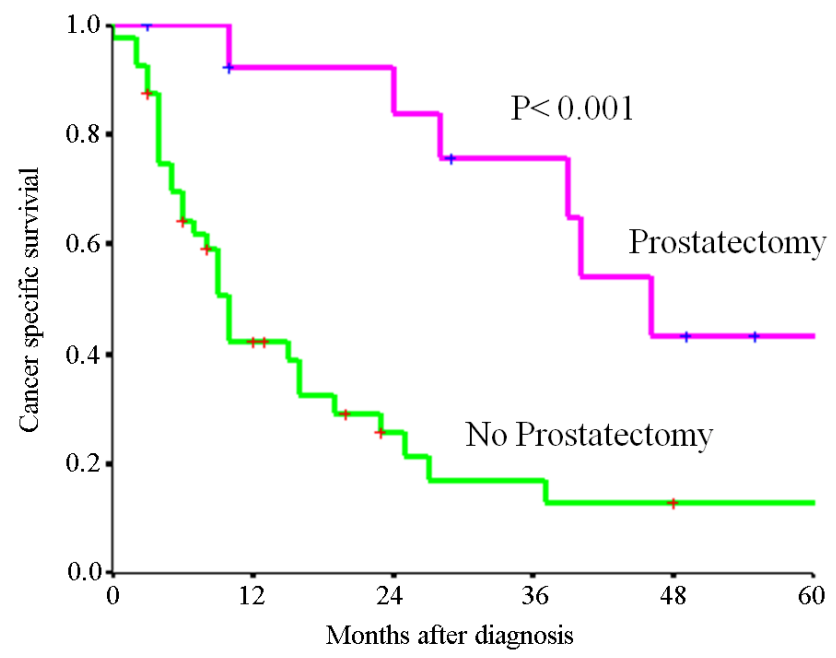

(c)

Figure 1. Kaplan-Meier survival curves for (a) Overall survival of entire cohort, (b) cancer specific survival of entire cohort, (c) cancer specific survival rate of patients by therapy. $P$ value shown for log-rank test between two groups.

patients with prostate cancer, due to the perception that the re-biopsy of these patients in the face of known prostate cancer is not warranted, especially in the case of elderly patients with advance stage disease. Inconsistent use of a standardized pathologic classification likely account for the conflicting results of many clinical series [4-8]. Numerous nomenclatures have been used to identify these tumors. Wick and Swanson preferred the unifying term "sarcomatoid carcinoma" due to the presumed origin from carcinoma with varying degrees of divergent differentiation, including sarcomatoid pattern [1]. In some case, carcinosarcoma of the prostate may be classified as metaplastic carcinoma, spindle-cell carcinoma, and malignant mixed mesodermal tumor. Based on above, some investigators postulate that the incidence and clinical impact of this subtype of prostate cancer are likely un- derestimated and underreported [19,20].

Clinically, the patients with prostate carcinosarcoma tend to be older man [4,7], and in roughly half of cases have a history of prostatic adenocarcinoma treated by radiation $[3,4,7]$. The tumors usually produce bladder outlet obstruction and often require repeated TURs to control local symptoms [7]. Serum PSA levels are usually lower than expected for the tumor volume [4]. Estrogen therapy may stimulate tumor growth, as suggested by the case reported by Hamlin and Lurid [16]. In contrast to adenocarcinoma of the prostate, carcinosarcoma is generally characterized by a rapid and lethal course [2, 3]. Therefore, it is critical to establish a correct histological diagnosis to avoid inadequate therapy such as orchiectomy and hormonal therapy. In the largest published series, 5- and 7-year survivals were 41 and 14\%, 
Table 3. Multivariate analyses of factors associated with cancer specific mortality in patients with carcinosarcoma of the prostate.

\begin{tabular}{cccc}
\hline Characteristics & Group & HR $(95 \% \mathrm{CI})$ & P value \\
\hline Age & & $0.98(0.94-1.02)$ & 0.41 \\
Ethnicity & White & 1.00 & \\
& Others & $0.73(0.23-2.29)$ & 0.73 \\
Marital status & Married & 1.00 & \\
& No & $0.89(0.08-2.07)$ & 0.79 \\
Diagnosis year & $1977-1994$ & 1.00 & \\
& $1995-2007$ & $0.76(0.38-7.22)$ & 0.81 \\
Prostatectomy & No & 1.00 & \\
& Yes & $0.12(0.04-0.40)$ & $<0.0001$ \\
Radiation & No & 1.00 & \\
& & $1.40(0.62-3.16)$ & 0.42 \\
Stage & Local/regional & 1.00 & \\
& Distant & $0.76(0.24-2.40)$ & \\
& Unknown & $0.41(0.05-3.56)$ & 0.42 \\
\hline
\end{tabular}

$\mathrm{HR}=$ Hazard ratio; $\mathrm{CI}=$ Confidence interval.

respectively [4]. The clinical characteristics of patients with carcinosarcoma of the prostate in this study consistent with previous published single institution studies, the cancer specific survival of this cohort of carcinosarcoma of the prostate was poor. In our study, the 1-, 3- and 5year survival rate of carcinosarcoma of the prostate were $50.4 \%, 26.2 \%$ and $11.4 \%$ (Figure 1(a)). Our findings confirmed prior reports that carcinosarcoma of the prostate is a highly aggressive subtype [2,4,7], carcinosarcoma of the prostate should be treated as a separate entity distinct from ordinary adenocarcinoma.

Owing to the rareness of carcinosarcoma of the prostate, there is no standard treatment for this disease. Transurethral resection of the prostate (TURP) carries the risk of incomplete tumor resection. Therefore, radical prostatectomy is the treatment of choice $[4,6,7,9,24]$. In this study, a multivariate survival analyses indentified prostatectomy as the sole independent factor associated with favorable cancer specific survival.

The prognosis of this tumor remains poor, even in those patients with resectable disease, local recurrence and/or metastasis rates were very high after radical surgery $[4,6]$. The overall 5 -year cancer specific survival rate after prostatectomy in our study population was only $21 \%$, suggesting a high risk of early dissemination at the time of prostatectomy. Accurate staging is an important for proper disease management. Curative therapy can only be performed in patients with localized prostate carcinoma. However, clinical staging to differentiate between localized and advanced disease stage appear to be unreliable [26-28]. Meticulous presurgical staging and selection of appropriate surgical candidates is essential.
Multimodality treatment including prostatectomy, pre- or postoperative radiation therapy, and neoadjuvant or adjuvant chemotherapy have been used in the management of carcinosarcoma of the prostate, but there are no standard treatment recommendations [2-4,7,11]. Patients with bulky disease may be treated with neoadjuvant (preoperative) chemotherapy with or without radiotherapy followed by an attempt for surgical resection.

Our findings should be interpreted within the limitations of the study. This is a nonrandomized study; therefore, selection bias might have been present because patients undergoing prostatectomy tend to be healthier. Although we adjusted for differences in demographic and clinical features, residual confounding might be present. In addition, our data did not allow us to examine surgical volume, hospital characteristic, receipt of chemotherapy, and patient comorbidities, all of which are factors presumed to influence survival in cancer patients [17]. However, the use of cancer specific survival rather than overall survival in our study has modified the limitation to some degree. Finally, sample size in our study may still not be enough to fully describe the factors that affect the incidence, treatment choice, and survival of this rare tumor.

\section{Conclusions}

In summary, carcinosarcoma of the prostate is a highly malignant neoplasm, occurring predominantly in elder males with an advanced stage at presentation and with poor prognosis. Radical prostatectomy is the only treatment modality that significantly improves survival. Accurate histological diagnosis, meticulous presurgical staging and selection of appropriate surgical candidates are essential. Emphasis on early diagnosis and detection and multimodality therapy of this disease is needed to improve the outcome for patients with this malignancy.

\section{Acknowledgements}

The authors acknowledge the efforts of the Applied Research Branch, Division of Cancer Prevention and Population Science, NCI; and the Surveillance, Epidemiology, and End Results (SEER) Program tumor registries. Interpretation and reporting of these data are solely the responsibility of the authors.

\section{REFERENCES}

[1] M. P. Wick and P. E. Swanson, "Carcinosarcomas: CurRent Perspectives and a Historical Review of Nosological Concepts," Seminars in Diagnostic Pathology, Vol. 10, No. 2, 1993, pp. 118-127.

[2] S. M. McGee, S. A. Boorjian and R. J. Karnes, "Carcinosarcoma of the Prostate Replacing the Entire Lower Geni- 
tourinary Tract," Urology, Vol. 74, No. 3, 2009, pp. 540541. doi:10.1016/j.urology.2009.04.057

[3] M. R. Wick, R. H. Young, R. Malvesta, D. S. Beebe, J. J. Hansen and L. P. Dehner, "Prostatic Carcinosarcomas: Clinical, Histologic and Immunohistochemicaldata on Two Cases with a Review of the Literature," American Journal of Clinical Pathology, Vol. 92, No. 2, 1989, pp. 131-139.

[4] P. A. Dundore, J. C. Cheville, A. G. Nascimento, G. M. Farrow and D. G. Bostwick, "Carcinosarcoma of the Prostate: Report of 22 Cases," Cancer, Vol. 76, No. 6, 1995 , pp. 1035-1042.

[5] A. A. Renshaw and S. R. Granter, "Metastatic Sarcomatoid, and PSA- and PAP-Negative Prostatic Carcinoma: Diagnosis by Fine Needle Aspiration," Diagnostic Cytopathology, Vol. 23, No. 3, 2000, pp. 199-201.

[6] N. Perez, M. Castillo, Y.Santos, D. Truan, R. Gutierrez, A. Franco, A. Palacin, J. A. Bombi , E. Campo and P. L. Fernandez, "Carcinosarcoma of the Prostate: Two Cases with Distinctive Morphologic and Immunohistochemical Findings," Virchows Arch, Vol. 446, No. 5, 2005, pp. 511-516. doi:10.1007/s00428-005-1239-x

[7] D. E. Hansel and J. I. Epstein, "Sarcomatoid Carcinoma of the Prostate: A Study of 42 Cases," American Journal of Surgical Pathology, Vol. 30, No. 10, 2006, pp. 13161321.

[8] V. S. Subramanian, M. Coburn and B. J. Miles, "Carcinosarcoma of the Prostate with Multiple Metastases: Case Report and Review of the Literature," Urologic Oncology, Vol. 23, No. 3, 2005, pp. 181-183. doi:10.1016/j.urolonc.2004.11.010

[9] C. G. Rogers, A. Parwani, A. Tekes, M. P. Schoenberg and J. I. Epstein, "Carcinosarcoma of the Prostate with Urothelial and Squamous Components," Journal of Urology, Vol. 173, No. 2, 2005, pp. 439-440. doi:10.1097/01.ju.0000149969.76999.7c

[10] T. Fukawa, K. Numata, M. Yamanaka, T. Miyamoto, Y. Kurokawa, H. O. Kanayama, S. Kagawa, M. Utsunomiya and M. Hirokawa, "Prostatic Carcinosarcoma: A Case Report and Review of Literature," International Journal of Urology, Vol. 10, No. 2, 2003, pp. 108-113. doi:10.1046/j.1442-2042.2003.00574.x

[11] S. E. Canfield, T. H. Gans, P. Unger and S. J. Hall, "Postradiation Prostatic Sarcoma: De Novo Carcinogenesis or Dedifferentiation of Prostatic Adenocarcinoma?" Techniques in Urology, Vol. 7, No. 4, 2001, pp. 294-295.

[12] E. Poblet, A. Gomez-Tierno and L. Alfaro, "Prostatic Carcinosarcoma: A Case Originating in a Previous Ductal Adenocarcinoma of the Prostate," Pathology Research and Practice, Vol. 196, No. 8, 2000, pp. 569-572.

[13] L. Thompson, B. Chang and S. H. Barsky, "Monoclonal Origins of Malignant Mixed Tumors (Carcinosarcomas). Evidence for a Divergent Histogenesis," American Journal of Surgical Pathology, Vol. 20, No. 3, 1996, pp. 277285.

[14] M. E. Ray, K. J. Wojno, N. S. Goldstein, K. B. Olson, R. B. Shah and K. A. Cooney, "Clonality of Sarcomatous and Carcinomatous Elements in Sarcomatoid Carcinoma of the Prostate," Urology, Vol. 67, No. 2, 2006, pp. 423.e5423.e8.

[15] L. J. Krastanova and J. C. Addonizio, "Carcinosarcoma of Prostate," Urology, Vol. 18, No. 1, 1981, pp. 85-88. doi:10.1016/0090-4295(81)90506-9

[16] W. B. Hamlin and P. K. Lund, "Carcinosarcoma of the Prostate: A Case Report," Journal of Urology, Vol. 97, No. 3, 1967, pp. 518-522.

[17] J. R. Haddad and E. C. Reyes, "Carcinosarcoma of the Prostate with Metastasis of Both Elements: Case Report," Journal of Urology, Vol. 103, No. 1, 1970, pp. 80-83.

[18] S. C. Quay and K. H. Proppe, "Carcinosarcoma of the Prostate: Case Report and Review of the Literature," Journal of Urology, Vol. 125, No. 3, 1981, pp. 436-438.

[19] K. Hokamura, T. Kurozumi, K. Tanaka and A. Yamaguchi, "Carcinosarcoma of the Prostate," Acta Pathologica Japonica, Vol. 35, No. 2, 1985, pp. 481-487.

[20] Surveillance, Epidemiology, and End Results (SEER) Program, Public-Use Data, National Cancer Institute, DCCPS, Surveillance Research Program, Cancer Statistics Branch. http://www.seer.cancer.gov/ about/expansion.html.

[21] SEER Summary Staging Manual, 2000. http://seer.cancer.gov/tools/ssm/

[22] E. L. Kaplan, and P. Meier, "Nonparametric Estimation from Incomplete Observations," Journal of the American Statistical Association, Vol. 53, 1958, pp. 457-481. doi: $10.2307 / 2281868$

[23] D. R. Cox, "Regression Models and Life-Tables," Journal of the Royal Statistical Society Series B, Vol. 34, 1972, pp. 187-220.

[24] J. L. Young, D. S. Finley and D. K. Ornstein, "Robotic-Assisted Laparoscopic Cystoprostatectomy for Prostatic Carcinosarcoma," Journal of the Society of Laparoendoscopic Surgeons, Vol. 11, No. 1, 2007, pp. 109-112.

[25] T. Y. Tseng, D. W. Sevilla, J. W. Moul and K. E. Maloney, "Prostatic Carcinosarcoma 15 Years after Combined External Beam Radiation and Brachytherapy for Prostatic Adenocarcinoma: A Case Report," Prostate Cancer and Prostatic Diseases, Vol. 9, No. 2, pp. 195-197. doi: $10.1038 /$ si.pcan. 4500870

[26] P. H. Lange and P. Narayan, "Understaging and Undergrading of Prostate Cancer. Argument for Postoperative Radiation as Adjuvant Therapy," Urology, Vol. 21, No. 2, 1983, pp. 113-118. doi:10.1016/0090-4295(83)90002-X

[27] B. R. Konety, R. Naraghi, W. Gooding, W. F. O’Donnell and R. R. Bahnson, "Evaluation of Computerized Tomography for Staging of Clinically Localized Adenocarcinoma of the Prostate," Urologic Oncology, Vol. 2, No. 1, 1996, pp. 14-19.

[28] R. E. Mitchell, M. H. Katz, J. M. McKiernan and M. C. Benson, "The Evaluation and Staging of Clinically Localized Prostate Cancer," Nature Clinical Practice Urology, Vol. 2, No. 8, 2005, pp. 356-357. 